\title{
Analysis of the lateral vibrations of an unbalanced Jeffcott rotor
}

\author{
Ali Alsaleh ${ }^{1}$, and Hassen M. Ouakad ${ }^{1}$ \\ ${ }^{1}$ King Fahd University of Petroleum \& Minerals, Mechanical Engineering Department, 31261, Dhahran, \\ Kingdom of Saudi Arabia.
}

\begin{abstract}
This paper examines experimentally and analytically the lateral vibrations of a Jeffcott rotor running at various unbalance states. Using a Bently Nevada RK-4 rotor kit, three states of eccentric mass unbalance were assumed in this study: $0.4 \mathrm{~g}, 0.8 \mathrm{~g}$ and $1.2 \mathrm{~g}$. Measurements of the startup data and the steady state data at rigid and flexible rotor condition were collected using a setup that mimics the vibration monitoring industrial practices. Lagrange method was assumed to construct a linear mathematical model of the investigated rotor, based on rigid rotor assumptions, that can predict analytically the lateral vibrations. The dynamic characteristics of the system, including the linearized bearing induced stiffness, were solely extracted from startup data. It was concluded that the developed twodegrees-of-freedom model was able to predict the lateral vibration at the rigid condition with an error around $5 \%$. Whereas it failed to predict the response at flexible condition with matching accuracy. Unlike the majority of the work done in this field where complex, nonlinear mathematical model were used to model real systems, this work validates the applicability of using simple mathematical models in predicting the response of a real rotorsystem with an acceptable accuracy.
\end{abstract}

\section{Introduction}

The literature presents good efforts on mathematically simulating the unbalance response of rotor systems with high accuracy. Zhou, et al. [1] presented a novel non-linear mathematical model based on Lagrange equations and the finite element method to simulate the response of a rotor when unbalance and other dynamic forces are applied. Sanches and Pederiva [2] worked on identifying the unbalance response in a rotor using correlation matrices and modal order reduction method. Those studies and many more, such as [3,4], were motivated mainly by the demand of machinery design firms to model the unbalance response of rotors with high accuracy. However, the case is different in machinery operation facilities were the need to such high accuracy is not often required, especially considering the testing requirement and the complexity of applying such techniques on running machines. Thus, this study comes with the intention to investigate the feasibility of using an analytical, linear and simple-to-use mathematical model to simulate the unbalance response of a rotor using only startup data collected with conventional vibration monitoring setup. If proven feasible, this model will help accelerating the decision-making process when it comes to dealing with unbalanced machines during operation.

\section{Experimental setup and procedure}

The experimental testing for this investigation, Figure 1, was conducted on Bently Nevada RK-4 single disk rotor kit. More details of the rotor-kit specifications are available in [5]. The setup, which is built to mimic Jeffcott rotor, was chosen as it is complex enough to mimic real dynamic response of rotating equipment, and yet easy enough to develop the mathematical model using conventional methods/laws: Newton's Laws, Energy Principle, etc. The measurements were collected using ADRE 408 data acquisition system of 24-bit resolution with $3300-8 \mathrm{~mm}$ proximity sensors installed $18.0 \mathrm{~cm}$ away from each bearing to avoid nodal points. Two tests were conducted in the study; a 
startup test to evaluate the system equivalent stiffness and damping, and a time-wave response test at constant speeds (1000 RPM and 3600 RPM) to check the validity of the mathematical model at a practical operation range. Three different unbalance states were simulated using $0.4,0.8$ and 1.2 grams eccentric masses.

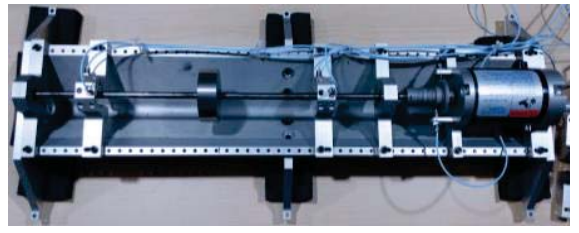

(a)

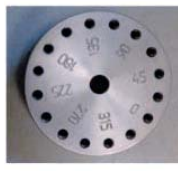

(b)

Figure 1. Photographs showing the Bently Nevada RK-4 rotor kit that consists of a stainless-steel rotating disk (800 grams of mass, $7.5 \mathrm{~cm}$ in diameter and $2.5 \mathrm{~cm}$ in thickness) with holes to attach eccentric masses at $30.0 \mathrm{~mm}$ mounted midway between two sleeve bearings with a clearance at each bearing of 5 mils and stainless-steel cylindrical shaft of $1.0 \mathrm{~cm}$ diameter and $50.0 \mathrm{~cm}$ between-bearing-length. As seen, the accelerometers are attached to the left-hand-side bearing (inboard) and from which the data were collected.

\section{Theory and solution approach}

\subsection{Assumptions and system degrees-of-freedom}

Considering the rotor kit seen in Figure 1-a, we can make the following set of assumptions,

1- The mass of the shaft is negligible compared to the mass of the disk (lumped-parameter system)

2- The shaft is elastic radially and rigid axially

3- $\quad$ The bearings are soft support

4- The dynamic properties of the two bearings are identical

5- The rotor rotation around axes perpendicular to the axis of rotation is negligible (static unbalance)

6- The lateral vibrations of the system are small

7- Damping is present in the form of viscous damping

The assumptions were either verified by inspection or made to simplify the development of the mathematical model. They all support the rigid rotor assumption neglecting the shear forces and the gyroscopic moment effects. We shall see the implications of making such assumptions on the accuracy of the mathematical model in the results section. Rigid bodies undergoing spatial motion can have generally a maximum of six degrees-of-freedom (DOF), three rotations and three displacements [6]. Assumptions \#2 and \#5, which are essentially constrains, reduce the number of DOF for this model from six into three, spin rotation and two lateral displacements. Specifying the rotational speed as a user-controlled parameter, the DOF get further reduced into two degrees only.

\subsection{Generalized coordinates and resulting equations of motion}

The experimental rotor can be modeled as seen in Figure 2. Point A and B represent the points of measurement were the proximity probes were installed, which correlate to the inboard and outboard bearings respectively. Since only one rotation is considered, a fixed-inertial reference frame, labeled as $\vec{x}, \vec{y}, \vec{z}$, is sufficient to fully describe the motion of the two points. In addition, assumption $\# 2$, \#4 and \#5 suggest that the displacement at point $A$ is identical to that at point $B$ since only spin rotation is considered. Therefore, the displacement along the $\vec{x}$ and $\vec{y}$ direction at point $A$, corresponding to the inboard bearing, are chosen as the generalized coordinates of the entire rotorsystem. 




Figure 2. Model representing the motion and kinetics of the studied rotor.

By properly identifying the forces acting on the system along the generalized coordinates, we arrive to the following equations of motion using Lagrange operator,

$$
\begin{aligned}
& M_{D} \ddot{x}_{A}+C_{e q x} \dot{x}_{A}+K_{e q} x_{A}=m e \omega^{2} \operatorname{Cos}(\omega t) \\
& M_{D} \ddot{y}_{A}+C_{e q y} \dot{y}_{A}+K_{e q_{y}} y_{A}=m e \omega^{2} \operatorname{Sin}(\omega t)
\end{aligned}
$$

where $M_{D}$ is the mass of the disk, $C_{\text {eqx }}$ and $C_{\text {eqy }}$ are the equivalent viscous damping coefficient along the $\vec{x}$ and $\vec{y}$ direction, and $K_{\text {eqx }}$ and $K_{\text {eqy }}$ are the equivalent stiffness along the $\vec{x}$ and $\vec{y}$ directions. The above equations of motion are in correspondence with what was developed by Yukio [7] of a similar 2 DOF rigid rotor-system.

\subsection{Dynamic system characteristics evaluation}

The unknown dynamic characteristics in Equations (1) and (2) are the system-equivalent damping and stiffness along each generalized coordinate. A practical method of evaluating the equivalent dynamic characteristics of a rotor system without getting into the details of system components (shaft, disk and bearings) is by analyzing startup data. In a bode plot, which is a typical presentation of startup data, the equivalent damping ratio $(\xi)$ can be evaluated using the half-power bandwidth method. Even though this method is strictly applicable to lightly damped materials $(\xi<0.05)$, it is still widely used with none lightly damped materials (Some modification needs to be considered) [8].

The system-equivalent stiffness of a rigid body along a generalized coordinate can also be evaluated using bode plot by identifying the resonance frequency. This frequency corresponds to the system natural frequency, which can then be used to evaluate the system-equivalent stiffness given that that mass of the system is known.

The analysis of the startup data can also lead to the evaluation of the bearing induced stiffness, which is often a desired outcome in the research field of rotor-dynamics due to their nonlinear effects. The overall stiffness of the system is induced by the shaft and the bearings. Since both stiffnesses are subjected to the same loading, they can be think of as two stiffnesses in series as seen in Figure 2. Shaft stiffness can be evaluated using the theory of strength of materials. The theory suggests that elastic elements like beams or shafts exhibits a static deflection when loaded. Therefore, they can be modeled as ideal springs. The stiffness of the shaft at x-distance away from the bearing can then be approximated by,

$$
K_{\text {shaft }}=\frac{P}{\delta_{\text {static }}} \approx \frac{48 E I}{\left[3 L^{2} x-4 x^{3}\right]}
$$

where $P$ is the loading applied on the shaft center, $E$ is the modulus of elasticity for the shaft, $I$ is the cross-sectional moment of inertia of the shaft and $L$ is the total length of the shaft between bearings. $E$ of steel shaft is $210 \mathrm{GN} / \mathrm{m}^{2}$ and $L$ of the shaft used in this study is $50 \mathrm{~cm}$. 


\section{Results and discussion}

A $1 \mathrm{X}$ bandpass filter was applied to all experimentally collected data to filter out the synchronous lateral vibrations. That is because the interest is to study the unbalance induced vibrations only. Figure 3 shows the bode plots of the startup data collected at each unbalance state for the inboard bearing (point $\mathrm{A}$ in Figure 2).
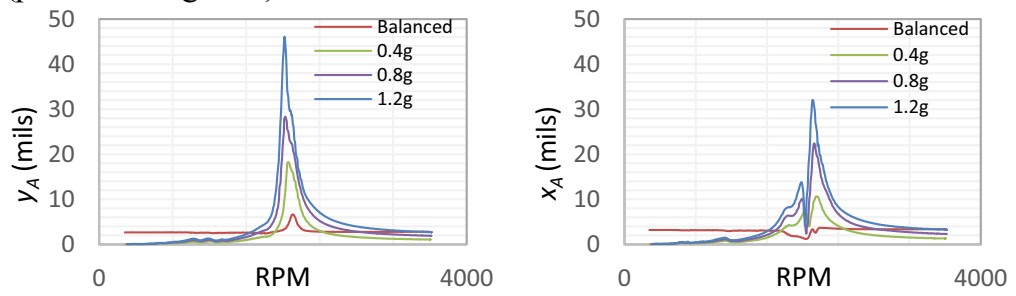

Figure 3. Bode plots showing the (1X) synchronous experimental lateral displacements as a function of rotation speed at the inboard bearing for each unbalance state.

Figure 3 shows the expected behavior of an unbalanced system subjected to increasing eccentric masses and it is well explained by Equation (1) and (2). What is not explained by the two equations and worth mentioning is the fact that resonance frequency for the three unbalance states seems to slightly decrease with increasing amplitude. This phenomenon is normal and has been observed before in similar studies, for example by Littler [9].

It is also observed that there is a variance in the amplitude of the vertical $(\vec{y})$ and horizontal $(\vec{x})$ vibrations at all states, where the vertical vibrations seem to be always higher. This supports the fact that bearings contribute significantly to the stiffness of the system. That is because, ideally a shaft of isotropic properties, if considered as the only source of stiffness in the system should lead to identical amplitude of vibrations along the lateral direction $(\vec{x}$ and $\vec{y})$. This realization was owed by Kelm to the oil film variation in sleeve bearings [10]. According to Kelm, a variant amplitude of vibrations along the lateral direction is commonly observed in machinery bearings and it is due to the variant oil pressure around the bearings. Variant oil pressure leads to variant stiffness properties along the lateral direction of the rotor. This notion was also supported by the work of Nicholas and Barret [11] and was taken into consideration when developing the mathematical model.

Using the half-power bandwidth method and by identifying the resonance frequency at each unbalance state as illustrated in subsection 3.3, the data in Figure 3 were used to evaluate the equivalent dynamic properties of the system. To account for the variation in the data at each unbalance state, the average value for each property was considered to develop the mathematical model as seen in Table 1.

Table 1. Average system dynamic characteristics based on the measurements at the inboard bearing.

\begin{tabular}{|c|c|c|c|c|}
\hline $\mathrm{GC}$ & $\zeta_{\text {eq }}$ & $K_{\text {eq }}(\mathrm{kN} / \mathrm{m})$ & $K_{\text {shaft }}(\mathrm{kN} / \mathrm{m})$ & $K_{\text {bearing }}(\mathrm{kN} / \mathrm{m})$ \\
\hline$\vec{y}$ & 0.0087 & 37.004 & 44.308 & 224.490 \\
\hline$\vec{x}$ & 0.0134 & 39.530 & 44.308 & 366.562 \\
\hline
\end{tabular}

The method presented in evaluating the bearing stiffness gives easy access to linearized bearing stiffness coefficient, which is often calculated in a nonlinear manner in literature. The values show that the bearing stiffness is higher than that of the shaft for both directions, and the stiffness along the horizontal direction $(\vec{x})$ is higher than that at the vertical direction $(\vec{y})$. This outcome matches that of studies like [12]. Based on the depicted system-equivalent properties, 
the mathematical model was developed. Figure 4 shows a sample of the simulated data at the $0.8 \mathrm{~g}$ unbalance state as compared to the experimental data.
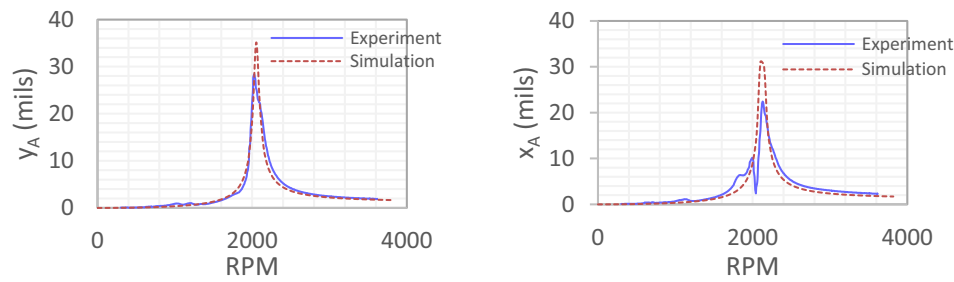

Figure 4. Bode plots showing the synchronous experimental lateral displacements and the simulated mathematical model response at the inboard bearing for the $0.8 \mathrm{~g}$ unbalance state.

To have a deeper insight on the accuracy of the mathematical model, the steady state response of the system for the same unbalance state at around 1000 RPM is considered as shown in Figure 5.


Figure 5. Timewave response showing the synchronous (1X) experimental lateral displacements and the simulated mathematical model response at the inboard bearing for the $0.8 \mathrm{~g}$ unbalance state at around 1000 RPM.

The error present in the simulated results is about $4 \%$ on both directions, taking the bearing clearances as a reference and not the experimental values. The reason behind choosing this error calculation method is that alarm and trip limits of machinery protection systems in the industrial practice are usually based on the mechanical clearances [13]. Thus, it is more representative to base the accuracy of the modal on the available mechanical clearances and not the experimental values. This outcome and accuracy of the mathematical model is expected at a speed below the critical speed due to the fact that the rotor is considered rigid at that speed range, which is in line with the assumptions made.

When it comes to flexible rotor behavior, Figure 6 shows that the amplitude of vibration increases and so does the error in the simulated results (maximum of $32 \%$ on both directions). This proves that the mathematical model does not capture the full dynamics of the system when the rotor is presumed flexible. This outcome is expected as the assumptions made did not account for the dynamic effects associated with the flexible rotor condition, such as the effect of gyroscopic moment. Table 2 summarizes the results for all tested unbalance states.


Figure 6. Timewave response showing the synchronous $(1 \mathrm{X})$ experimental lateral displacements and the simulated mathematical model response at the inboard bearing for the $0.8 \mathrm{~g}$ unbalance state at around 3600 RPM. 
Table 2. Inboard bearing experimental and simulated peak-to-peak amplitudes.

\begin{tabular}{|c|c|c|c|c|c|c|c|c|c|c|}
\hline \multirow[t]{2}{*}{$\sim \mathrm{RPM}$} & \multirow[t]{2}{*}{$\mathrm{GC}$} & \multicolumn{3}{|c|}{$\begin{array}{l}\text { Experimental results } \\
\text { peak-to-peak amplitude } \\
\text { (mils) }\end{array}$} & \multicolumn{3}{|c|}{$\begin{array}{c}\text { Simulation results } \\
\text { peak-to-peak amplitude } \\
\text { (mils) }\end{array}$} & \multicolumn{3}{|c|}{ Error\% $\%$} \\
\hline & & $4 \mathrm{~g}$ & $8 \mathrm{~g}$ & $1.2 \mathrm{~g}$ & $4 \mathrm{~g}$ & $8 \mathrm{~g}$ & $1.2 \mathrm{~g}$ & $4 \mathrm{~g}$ & $8 \mathrm{~g}$ & $1.2 \mathrm{~g}$ \\
\hline \multirow{2}{*}{1000} & $\vec{y}$ & 0.614 & 0.970 & 1.137 & 0.396 & 0.792 & 1.148 & $4 \%$ & $4 \%$ & $0 \%$ \\
\hline & $\vec{x}$ & 0.569 & 0.880 & 1.295 & 0.363 & 0.726 & 1.053 & $4 \%$ & $3 \%$ & $5 \%$ \\
\hline \multirow{2}{*}{3600} & $\vec{y}$ & 1.074 & 1.891 & 2.719 & 1.749 & 3.486 & 5.227 & $14 \%$ & $32 \%$ & $50 \%$ \\
\hline & $\vec{x}$ & 1.339 & 2.276 & 2.978 & 1.809 & 3.603 & 5.403 & $9 \%$ & $27 \%$ & $48 \%$ \\
\hline
\end{tabular}

The outboard bearing measurements for the rigid-rotor shows very similar error values to the inboard ones for the rigid-rotor case. This validate the assumption of identical vibrations amplitude at both bearings for rigid rotors. As for the flexible rotor condition and similar to the inboard bearing results, the mathematical model also fails to capture the vibration amplitude with a maximum error of $51 \%$.

\section{Conclusion}

This study investigates the feasibility of mathematically modeling a single mass Jeffcott rotor to simulate the system lateral vibrations when running at various unbalance states. The mathematical model was developed based on Lagrange equations and rigid rotor assumptions. Using only Startup data to depict the system equivalent dynamic characteristics in a linear fashion, the linear model was found to accurately predict the real system response, in the rigid case only, with a maximum error of 5\%. This realization proves that relying on a linear and easy-to-use mathematical model can yield fast, and more importantly, reliable results in the field of rigid machinery rotors. As for the fixable-rotor condition, the model needs to be modified to account for the gyroscopic moment effects, the shear forces and the other dynamical effects that are associated with flexible rotor dynamics. For future considerations, it is suggested that this model get further tested on real plant machines, operating at the rigid rotor region, to study the effect of the rotor complexity and the flow induced loads on the validity of the model.

\section{References}

[1] W. J. Zhou, X. S. Wei, X. Z. Wei, L. Q. Wang, J. Zhejiang Univ. Sci. A 15, 39 (2014)

[2] F. D. Sanches, R. Pederiva, Mech. Mach. Theory, 101, 209 (2016)

[3] H.K. Yadav, S. H. Upadhyay, S. P. Harsha, Procedia Eng. 64, 593 (2013)

[4] M. R. Shad, G. Michon, A. Berlioz, Appl. Math. Model, 35, 2145 (2011)

[5] "RK-4 Rotor Kit, Bently Nevada Asset Condition Monitroing"

[6] J. Ginsperg, "Advanced Engineering Dynamics" Second Edition. CUP, (1998)

[7] Y. Toshio, I. Yukio, R. Kirk, Appl. Mech. Rev. 55 (2002)

[8] J. Butterwoth, J. H. Lee, B. Davidson. 13th World Conference on Earthquake Engineering. (2004)

[9] J. D. Littler, J Wind Eng. Ind. Aerod., 57, 179 (1995)

[10] Kelm, R., D., "Journal Bearing Analysis”, Vibration Institute Publications.

[11] J. C. Nicholas, L. E. Barrett, ASLE Transactions, 29, 329 (1986)

[12] Z. Abduljabbar, M. M. ElMadany, and E. Al-Bahkali, CSI, 65, 849 (1997)

[13] “Machinery Protection Systems, API 670”, American Petroleum Institute Standard, (2014) 